\title{
IL-6 Improves the Nitric Oxide-Induced Cytotoxic CD8+ T Cell Dysfunction in Human Chagas Disease
}

\author{
Liliana Maria Sanmarco', Laura Marina Visconti' ${ }^{2}$ Natalia Eberhardt ${ }^{1}$, \\ Maria Cecilia Ramello', Nicolás Eric Ponce ${ }^{1}$, Natalia Beatriz Spitale ${ }^{2}$, Maria Lola Vozza ${ }^{2}$, \\ Germán Andrés Bernardi², Susana Gea ${ }^{1}$, Angel Ramón Minguez ${ }^{2}$ and Maria Pilar Aoki ${ }^{1 *}$ \\ ${ }^{1}$ Centro de Investigaciones en Bioquímica Clínica e Inmunología CIBICI-CONICET, Facultad de Ciencias Químicas, \\ Universidad Nacional de Córdoba, Córdoba, Argentina, ${ }^{2}$ Hospital Nuestra Señora de la Misericordia del Nuevo Siglo, \\ Córdoba, Argentina
}

Reactive oxygen and nitrogen species are important microbicidal agents and are also involved in lymphocyte unresponsiveness during experimental infections. Many of the biological effects attributed to nitric oxide are mediated by peroxynitrites, which induce the nitration of immune cells, among others. Our group has demonstrated that nitric oxide is involved in the suppressive activity of myeloid-derived suppressor cells in Trypanosoma cruzi-infected mice, with a higher number of CD8+ T cells suffering surface-nitration compared to uninfected controls. Studying the functional and phenotypic features of peripheral CD8+ T cells from chagasic patients and human cells experimentally infected with $T$. cruzi, we found that different regulatory mechanisms impaired the effector functions of $T$ cytotoxic population from seropositive patients. Peripheral leukocytes from chagasic patients showed increased nitric oxide production concomitant with increased tyrosine nitration of CD8+ T cells. Additionally, this cytotoxic population exhibited increased apoptotic rate, loss of the TCR -chain, and lower levels of CD107a, a marker of degranulation. Strikingly, IL-6 stimulation of in vitro-infected peripheral blood mononuclear cells obtained from healthy donors, blunted T. cruzi-induced nitration of CD3+CD8+ cells, and increased their survival. Furthermore, the treatment of these cultures with an IL-6 neutralizing antibody increased the percentage of $T$. cruzi-induced CD8+ $T$ cell nitration and raised the release of nitric oxide. The results suggest that the under-responsiveness of cytotoxic $T$ cell population observed in the setting of long-term constant activation of the immune system could be reverted by the pleiotropic actions of IL-6, since this cytokine improves its survival and effector functions.

Accepted: 08 December 2016

Published: 23 December 2016

Citation:

Sanmarco LM, Visconti LM, Eberhardt N, Ramello MC, Ponce NE, Spitale NB, Vozza ML, Bernardi GA,

Gea S, Minguez AR and Aoki MP

(2016) IL-6 Improves the Nitric Oxide-Induced Cytotoxic CD8+ T Cell

Dysfunction in Human

Chagas Disease.

Front. Immunol. 7:626.

doi: 10.3389/fimmu.2016.00626
Keywords: tyrosine nitration, peroxynitrite, CD39, CD73, oxidative stress, Trypanosoma cruzi infection

\section{INTRODUCTION}

$\mathrm{CD} 8+\mathrm{T}$ cells play a critical role in the immunity against intracellular pathogens, including the protozoan parasite Trypanosoma cruzi, the causative agent of Chagas cardiomyopathy (1). Chagas disease is characterized by two distinct phases: the acute phase, which lasts several weeks and is characterized by non-specific symptoms; and the chronic phase, lasting lifelong. The host's ability to control T. cruzi infection is substantial, but not fully effective, since most infected individuals tightly 
limit parasite numbers but fail to completely clear the infection due to diverse and fascinating immune evasion processes (2). Indeed, parasite persistence at low levels into target cells is the hallmark of the indeterminate or asymptomatic chronic phase (3). Up to $40 \%$ of infected individuals develop progressive heart disease leading to an end-stage dilated cardiomyopathy.

One key defense mechanism against T. cruzi is exerted by nitric oxide (NO), which is produced by inducible NO synthase, among other enzymes, present in monocytes/macrophages and cardiomyocytes $(4,5)$. The parasite triggers activation of macrophage NADPH oxidase, resulting in a continuous production of superoxide anion $\left(\mathrm{O}_{2}^{-}\right)$and also stimulates infected macrophages to produce different amounts of NO. Despite its importance as a microbicidal agent at high levels, persistent low levels of NO have been involved in the establishment and maintenance of lymphocyte unresponsiveness in different experimental models of parasite infections (6-8). These mechanisms illustrate a clear parasite evasion strategy. Moreover, NO induces apoptosis of many different cell types in vitro and in vivo $(9,10)$. We found that IL-6 regulates inflammasome activation and, consequently, IL-1 $\beta$-induced NO production in a murine model of T. cruzi infection. The anti-inflammatory action of IL- 6 seems to be central for controlling local and systemic oxidative stress, promoting cellular rescue of apoptosis, and protecting infected IL-6-deficient mice against death (unpublished observation). This could be a novel mechanism that regulates NO release in the setting of T. cruzi infection.

The presence of low levels of NO rapidly initiates a reaction with the superoxide anion generating peroxynitrites that induce the nitration of surface proteins on T cells $(11,12)$. It is widely accepted that reactive nitrogen species (NO and peroxynitrites) contribute to the immunosuppressive attitude of myeloid-derived suppressor cells (MDSCs), a heterogeneous cell population associated with tumors, infectious, and inflammatory diseases. Our group has demonstrated that MDSCs during acute T. cruzi experimental infection were able to inhibit $\mathrm{T}$ cell proliferation in vitro (13). Furthermore, we also observed a higher number of splenic CD8+ T cells suffering surface nitration in infected mice compared to uninfected controls.

Chronic exposure to antigens may cause functional defects in pathogen-specific $\mathrm{T}$ cells. It has been reported that individuals with more severe clinical disease have a significantly lower frequency of T. cruzi-specific CD8+IFN $\gamma+$ T cells than subjects in the asymptomatic stage of the infection (14). This impairment in T. cruzi-specific CD8+ T cell responses was associated with an increased frequency of fully differentiated memory cells and an increased rate of apoptosis in the total peripheral CD8+ $\mathrm{T}$ cell population. The results reveal a progressive exhaustion in the parasite-specific cytotoxic $\mathrm{T}$ cell compartment in patients with long-term T. cruzi infection. However, during persistent infection, the chronic exposure to an inflammatory microenvironment may also contribute to the impairment of CD8+ T cell responses, resulting in a less efficient control of the pathogen and promoting its persistence.

These findings prompted us to investigate the frequency and functionality of circulating CD8+ T cells from patients with chronic Chagas disease, with particular focus on the molecular mechanisms triggered by IL- 6 associated with cytotoxicity and NO-induced cell death. In agreement with previous reports in seropositive patients with Chagas disease, we found a substantial reduction in the total peripheral $\mathrm{T}$ cell compartment at the expense of CD8+ T cells (14). Additionally, we observed increased NO-producing leukocytes concomitant with increased nitration of CD8+ T cells and impaired cytotoxic functionality of T cells. Strikingly, IL- 6 prevented the nitration of CD8+ T cells and increased their survival when healthy donor peripheral blood mononuclear cells (PBMCs) were infected in vitro. In this context, IL- 6 led to a decrease in IL- $1 \beta$ levels. The results suggest that the under-responsiveness of the whole cytotoxic T cell population from chagasic patients could be reverted by the pleiotropic actions of IL-6, since this cytokine functions as a survival factor for CD8+ cells and improves the cytotoxic T cell functionality in the setting of long-term constant activation of the immune system.

\section{MATERIALS AND METHODS}

\section{Ethics Statement}

Subjects were recruited at the "Hospital Nuestra Señora de la Misericordia” (HNSM) from Córdoba-Argentina. All studies were conducted according to the principles expressed in the Declaration of Helsinki. Signed informed consent was obtained from all donors before inclusion in the study. Venous blood was drawn from 46 non-chagasic and chagasic subjects (age 25-60 years) using a protocol that was reviewed and approved by the Comité Institucional de Ética de la Investigación en Salud del Adulto, Ministerio de Salud de la Provincia de Córdoba (Acta number 194/2014, Argentina). T. cruzi infection was determined by a combination of indirect hemagglutination (IHA) and enzyme-linked immunosorbent assay (ELISA) performed in the laboratory of HNSM. Subject positive on these two tests were considered infected. Chronic chagasic patients $(n=22)$ were evaluated clinically and by electrocardiogram and chest X-ray. The uninfected control group $(n=24)$ consisted of age-matched individuals were serologically negative for T. cruzi. All donors with chronic or inflammatory pathology or erythrocyte sedimentation rate $>30 \mathrm{~mm}$ or white blood cells count $<4,000$ or $>10,000$ / $\mathrm{mm}^{3}$ were excluded from the study.

\section{Blood Collection}

Approximately $15 \mathrm{~mL}$ of blood was drawn from each individual by venipuncture and placed into heparinized tubes (Vacutainer, BD Bioscience). An aliquot of $50 \mu \mathrm{L}$ of total peripheral blood was stained for FACS analysis and subjected to ACK lysing buffer to remove erythrocytes. PBMCs were isolated through density gradient centrifugation using Ficoll-Hypaque PLUS (GE Healthcare Bioscience) and resuspended in RPMI 1640 (Gibco) supplemented with $10 \%$ heat-inactivated FCS (Natocor).

\section{Ex Vivo Flow Cytometry}

Peripheral blood was lysed with ACK lysing buffer to remove erythrocytes, and $01 \times 10^{6}$ cells were blocked with Fc block and stained with anti-human CD3 Alexa 488 or PerCP, anti-human 
CD4 Alexa 647, anti-human CD8 PECy7, anti-human CD39 biotin and streptavidin APC, anti-human CD73 PE (eBioscience and Biolegend), anti-nitrotyrosine (Sigma-Aldrich), and antirabbit Alexa 647 or CD20 PECy7 (Biolegend). The intracellular expression of $\zeta$-chain was analyzed with anti-human TCR $\zeta$ PE antibody (Beckman Coulter). Stained cells were analyzed by flow cytometry (FACS Canto II, Becton Dickinson), with cellular debris being excluded from the analysis. Data were analyzed using the FlowJo software.

\section{Cell Viability Measurement}

Leukocytes from peripheral blood were stained with anti-human CD3 Alexa 488, anti-human CD4 Alexa 647, anti-human CD8 PECy7 or APCCy7, anti-human CCR7 Alexa 488, anti-human CD45RA PECy7, and labeled with $5 \mu \mathrm{L}$ of Annexin VPE (BD Pharmingen) for 15 min on ice. Before acquisition, the cells were stained with 7-AAD (BD Bioscience). A minimum of 300,000 events for each condition were analyzed by flow cytometry. Staining of peripheral blood T cells with antibodies to CD45RA and CCR7 reveals four cells subsets: naïve T cells are CD45RA+CCR7+, central memory cells are CD45RA-CCR7+, effector memory (EM) cells are CD45RA-CCR7-, and terminally differentiated effector memory (EMRA) are CD45RA+CCR7- (15).

To determine $\mathrm{Bcl}-2$ expression, PBMCs stained for CD3 PerCP, CD8, CD4, CCR7, and CD45RA surface expression were permeabilized with FOXP3 staining buffer set (eBioscience) and labeled with anti-Bcl-2 rabbit (Cell Signaling) and then with anti-rabbit Alexa 488 or anti-rabbit Alexa 647. Stained cells were analyzed by flow cytometry.

\section{CD8+ T Cell Functionality}

Peripheral blood mononuclear cells were cultured with antiCD107a PE (Biolegend), monensin, brefeldin A, phorbol 12-myristate 13-acetate (PMA), and ionomycin (Sigma) for $4 \mathrm{~h}$, then stained with anti-IFN $\gamma$ FITC, anti-TNF APC, and anti-IL-2 APC-Cy7 (Biolegend or eBioscience), and analyzed by flow cytometry.

To evaluate the effect of TCR-dependent activation, PBMCs were cultured with anti-CD3 $(1 \mu \mathrm{g} / \mathrm{mL})$ and anti-CD28 $(0.5 \mathrm{mg} /$ $\mathrm{mL}$ ). After $72 \mathrm{~h}, \mathrm{PBMCs}$ were cultured with monensin, brefeldin $\mathrm{A}$, and anti-CD107a PE for $6 \mathrm{~h}$ and then stained with anti-human CD8 APCCy7, anti-NT rabbit and anti-rabbit Alexa 647, antiIL-2 PECy7, anti-TNF PerCPCy5.5, or anti-IFNy PerCPCy5.5.

\section{CD8+ T Cell Exhaustion}

Peripheral blood mononuclear cells were cultured with anti-CD3 $(1 \mu \mathrm{g} / \mathrm{mL})$ and anti-CD28 $(0.5 \mathrm{mg} / \mathrm{mL})$. After $72 \mathrm{~h}$, the cells were stained with anti-human CD8 APCCy7, anti-Tim3 PerCPCy5.5, anti-PD1 PECy7, anti-CTLA4 PE, anti-CCR7 Alexa 488, and anti-NT rabbit and anti-rabbit Alexa 647.

\section{Measurement of Reactive Oxygen and Nitrogen Species}

The nitrite/nitrate content, indicative of NO production, was monitored by the Griess reagent assay (16). In 96-well plates, plasma samples were mixed with $50 \mu \mathrm{L}$ of Griess reagent, consisting of $1 \%$ sulfanilamide in $5 \%$ phosphoric acid and $0.1 \%$ $\mathrm{N}$-(1-napthyl) ethylenediamine dihydrochloride [1:1 ratio (vol/ vol)], and incubated for $10 \mathrm{~min}$. The change in absorbance was monitored at $545 \mathrm{~nm}$ (standard curve, 0-200 $\mu \mathrm{mol}$ sodium nitrite).

The production of ROS and NO was evaluated using the molecular probes: $\mathrm{H}_{2} \mathrm{DCF}-\mathrm{DA}(10 \mu \mathrm{M}$, Invitrogen Inc.) and DAF-FM DA (10 $\mu \mathrm{M}$, Molecular Probes, Inc.), respectively. All samples were acquired on a FACS Canto II cytometer and then analyzed using the Flow Jo software.

\section{Quantification of Cytokines}

Plasma samples were analyzed for TNF, IL-6, IL-10, IFN $\gamma$, and IL-4 by using bead-based immunoassays and flow cytometry, according to the manufacturer's instructions (LegendPlex-Biolegend). In cultured supernatant, IL- $1 \beta$ levels were measured by ReadySET-Go ELISA kit from eBioscience.

\section{Culture of PBMCs and Parasites}

Vero cell monolayers were infected with trypomastigote forms of T. cruzi Tulahuen strain for $3 \mathrm{~h}$ and then washed and maintained in RPMI (Gibco Invitrogen Corporation) at $37^{\circ} \mathrm{C}$ in a $5 \% \mathrm{CO}_{2}$ atmosphere. After 7 days, the parasites were collected from the supernatant of infected cells and harvested by centrifugation at 4,400 rpm for $5 \mathrm{~min}$.

Peripheral blood mononuclear cells from non-chagasic donors were cultured with T. cruzi Tulahuen trypomastigotes (1:1 rate) for $3 \mathrm{~h}$, then the cells were washed and cultured with recombinant bioactive IL-6 $(20 \mathrm{ng} / \mathrm{mL})$ or with anti-IL-6 $(2 \mu \mathrm{g} / \mathrm{mL})$ (Biolegend) or anti-IL-6 plus anti-IL-1 $\beta(2 \mu \mathrm{g} / \mathrm{mL})$, or maintained in medium alone for $48 \mathrm{~h}$. Then, culture supernatants were evaluated for IL- $1 \beta / \mathrm{NO}$ levels, and the cells were analyzed for NT staining or NO production by flow cytometry. In addition, peripheral blood from chagasic patients and control donors $(250 \mu \mathrm{L})$ were cultured with 7,500 trypomastigotes with or without IL-6 $(20 \mathrm{ng} / \mathrm{mL})$ or maintained in medium for $24 \mathrm{~h}$ and then cells were harvested to evaluate NO and ROS production and NT by flow cytometry.

\section{Statistical Analysis}

Statistical analysis was performed with GraphPad Prism 5.0 software by using parametric or non-parametric paired $t$ test according to data distribution. $p$ Values $<0.05$ were considered significant.

\section{RESULTS}

\section{Chagasic Patients Showed a Lower Number of Peripheral Blood CD8+ T Lymphocytes}

A total of 46 peripheral blood samples from chagasic and non-chagasic donors of both sexes, collected from people living in Cordoba (Argentina) were analyzed for T. cruzi-specific antibodies by ELISA and IHA (Table 1). The median value for anti-T. cruzi antibody titers detected by IHA was 1/256 (local 
cutoff titer 1/32). Only one patient showed a complete rightbundle branch block, left anterior hemiblock, and left atrial enlargement, clinical features of chagasic heart disease. Cordoba is considered by the Argentine Ministry of Health to be at high

TABLE 1 | Description of subject groups.

\begin{tabular}{lcc}
\hline & $\begin{array}{c}\text { Non-chagasic } \\
\text { donors }(\boldsymbol{n}=\mathbf{2 4})\end{array}$ & $\begin{array}{c}\text { Chagasic } \\
\text { patients }(\boldsymbol{n}=\mathbf{2 2})\end{array}$ \\
\hline $\begin{array}{l}\text { Age (years old) } \\
\text { Range }\end{array}$ & $25-60$ & $25-48$ \\
Median & 30 & 32 \\
Gender & & \\
Female & $n=16$ & $n=17$ \\
Male & $n=8$ & $n=5$ \\
Clinical evaluation & & \\
Electrocardiographic changes & $\mathrm{NE}$ & $n=1^{\mathrm{a}}$ \\
Echocardiographic changes & $\mathrm{NE}$ & $n=1^{\mathrm{b}}$ \\
Chest X-rays abnormalities & $\mathrm{NE}$ & $n=0$
\end{tabular}

${ }^{a}$ Complete right-bundle branch block and left anterior hemiblock.

'Left atrial enlargement.

NE, not evaluated. risk for vector transmission, since there is a reemergence of this infection route by an increase in house infestation and a high seroprevalence in vulnerable groups (17).

TABLE 2 | Peripheral blood lymphocyte subpopulations.

$\begin{array}{ccc}\text { Non-chagasic } & \text { Chagasic } & p \text {-Value } \\ \text { donors }(n=15) & \text { patients }(n=8) & \end{array}$

\section{Lymphocyte subsets in peripheral blood}

\begin{tabular}{|c|c|c|c|}
\hline CD3+ (\%) & $58.6 \pm 3.9$ & $43.2 \pm 5.3$ & $p=0.03^{\star}$ \\
\hline $\begin{array}{l}\mathrm{CD} 3+/ \mu \mathrm{L} \\
\text { (absolute number) }\end{array}$ & $1,139 \pm 102$ & $754 \pm 135$ & $p=0.04^{*}$ \\
\hline CD3+CD4+ (\%) & $58.7 \pm 3.7$ & $57.6 \pm 2.0$ & $p=0.84$ \\
\hline $\begin{array}{l}\mathrm{CD} 3+\mathrm{CD} 4+/ \mu \mathrm{L} \\
\text { (absolute number) }\end{array}$ & $600 \pm 72$ & $535 \pm 65$ & $p=0.59$ \\
\hline $\mathrm{CD} 3+\mathrm{CD} 8+(\%)$ & $39.9 \pm 3.5$ & $30.74 \pm 2.2$ & $p=0.04^{\star}$ \\
\hline $\begin{array}{l}\mathrm{CD} 3+\mathrm{CD} 8+/ \mu \mathrm{L} \\
\text { (absolute number) }\end{array}$ & $381 \pm 56$ & $193 \pm 32$ & $p=0.049^{\star}$ \\
\hline CD20+ (\%) & $14.1 \pm 1.5$ & $12.3 \pm 1.6$ & $p=0.44$ \\
\hline $\begin{array}{l}\mathrm{CD} 20+/ \mu \mathrm{L} \\
\text { (absolute number) }\end{array}$ & $305 \pm 40$ & $218 \pm 32$ & $p=0.16$ \\
\hline
\end{tabular}

Values are expressed as mean value $\pm S E$.

${ }^{*} p<0.05$, significant.

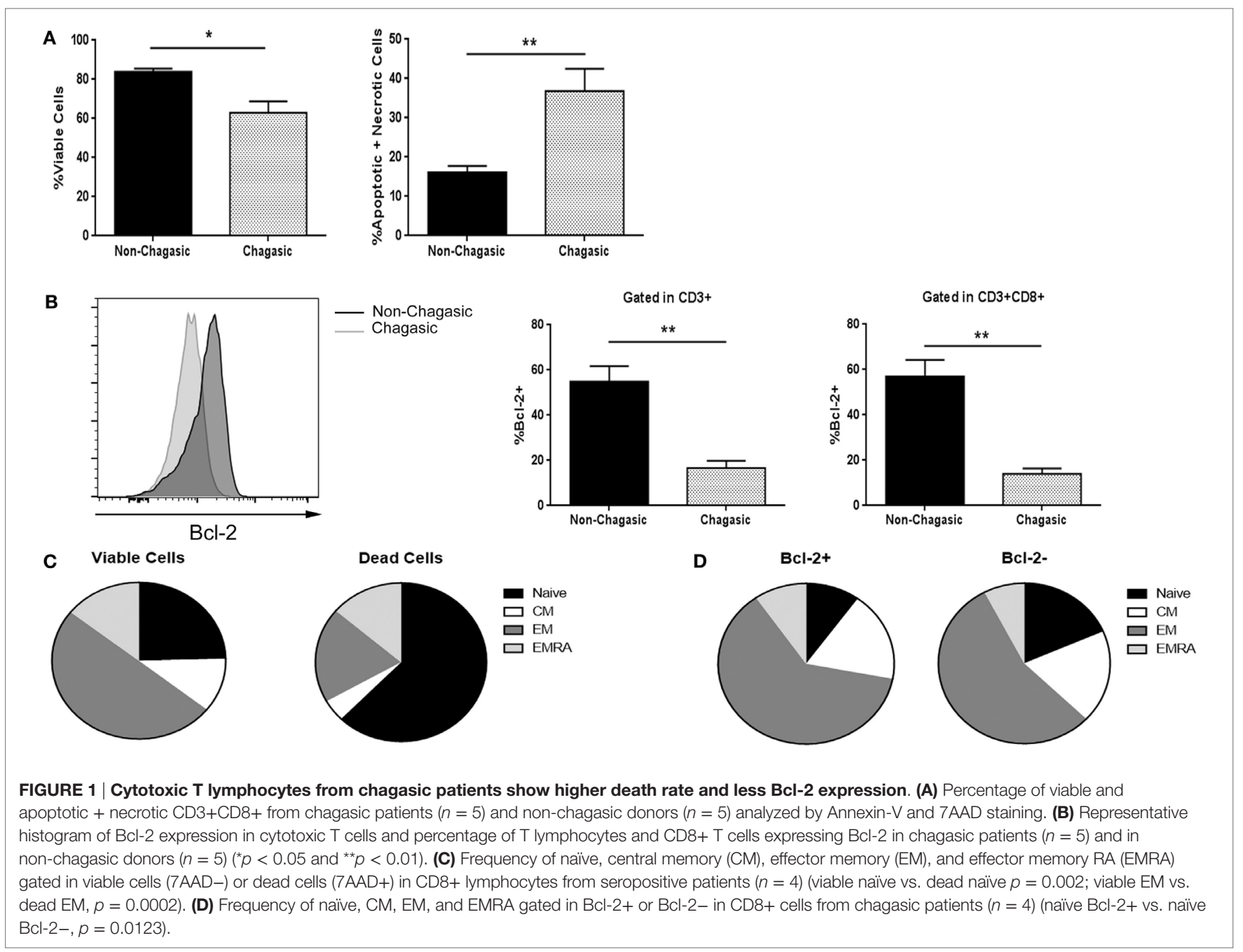



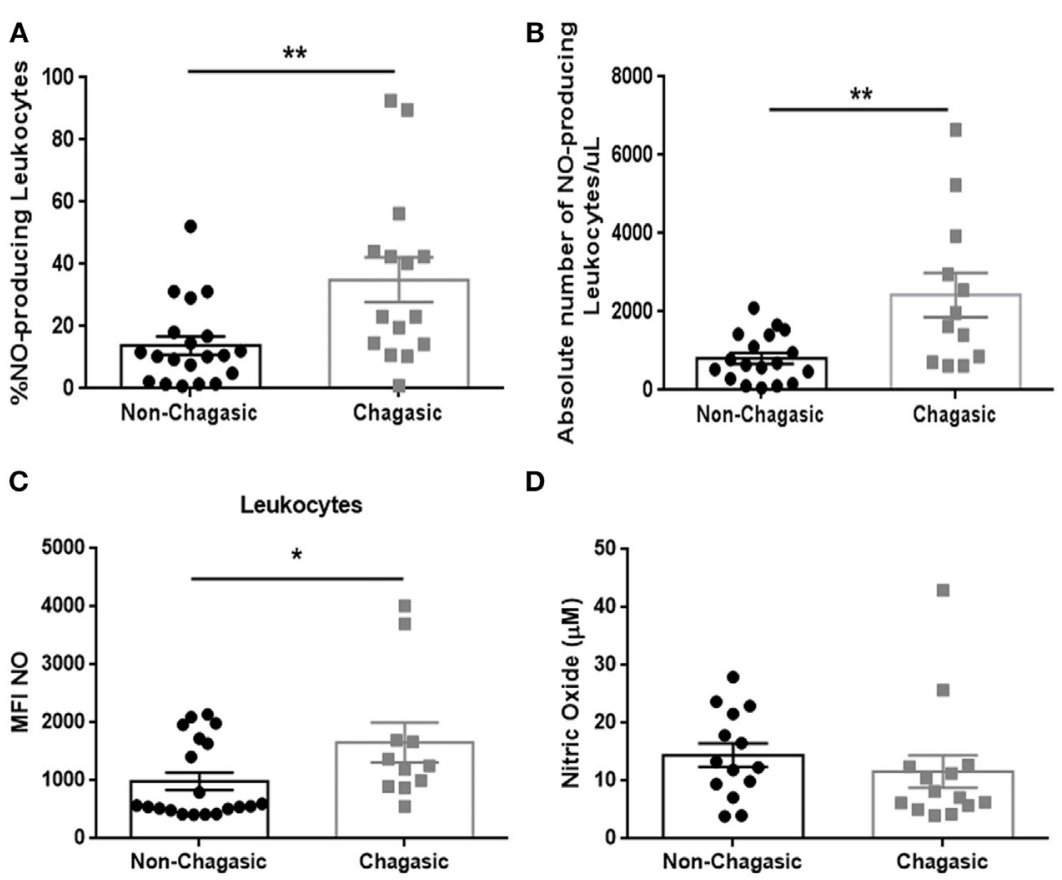

FIGURE 2 | Chagasic patients exhibit a higher number and frequency of nitric oxide-producing leukocytes. (A) Percentage and (B) absolute number of nitric oxide (NO)-producing leukocytes from chagasic patients $(n=15)$ and non-chagasic donors $(n=19)$. (C) Mean fluorescence intensity of NO in leukocytes from chagasic patients $(n=11)$ and non-chagasic donors $(n=20)$. (D) Serum nitric oxide levels in seropositive patients $(n=14)$ and seronegative donors $(n=14)$ $\left({ }^{\star} p<0.05\right.$ and $\left.{ }^{* *} p<0.01\right)$.

Seropositive patients with Chagas disease showed a diminished percentage and absolute number of total peripheral T lymphocytes $(\mathrm{CD} 3+)$ compared to seronegative donors. Although no differences were observed between non-chagasic and chagasic patients in the percentage and absolute number of $\mathrm{T}$ helper cells (CD3+CD4+), the percentage and absolute number of cytotoxic $\mathrm{T}$ cells $(\mathrm{CD} 3+\mathrm{CD} 8+)$ were significantly lower in seropositive than in seronegative patients (Table 2).

No differences were observed in the percentage and absolute number of B lymphocytes (CD20+) between the analyzed groups (Table 2).

\section{Cytotoxic T Lymphocytes from Chagasic Patients Showed Higher Cell Death and Less Bcl-2 Expression}

Through Annexin V and 7-AAD staining, we found that fresh explanted CD8+ T lymphocytes from chagasic patients showed a diminished percentage of viable cells, and concomitantly, a higher rate of apoptotic and necrotic cells compared to nonchagasic donors (Figure 1A). In agreement, the expression of the anti-apoptotic protein $\mathrm{Bcl}-2$ was significantly higher in cytotoxic lymphocytes from non-chagasic than from chagasic patients (Figure 1B). Among cytotoxic T cells from Chagas patients, naïve CD8 T cells were the phenotype most susceptible to die (viable cells vs. dead cells: $24.53 \pm 6.11$ vs. $62.48 \pm 3.95 \%$ ). In contrast, EM cytotoxic $\mathrm{T}$ cells showed significantly increased survival rate (viable cells vs. dead cells: $49.75 \pm 3.06$ vs. $19.48 \pm 2.45 \%$ )
(Figure 1C). In accordance, the frequency of naïve cells negative for intracellular Bcl-2 expression was significantly lower than naïve cells positive for the expression of this anti-apoptotic protein (Bcl-2+ vs. Bcl-2-: $9.95 \pm 0.87$ vs. $17.67 \pm 2.07$ ) (Figure 1D). Furthermore, the percentage of EM CD8 T cells was higher in Chagas subjects in comparison with seronegative donors (Figure S1 in Supplementary Material).

\section{Nitric Oxide-Producing Leukocytes Were Increased in Chagasic Patients}

Seropositive patients showed an increased percentage and absolute number of NO-producing leukocytes (Figures 2A,B), with higher production of NO per leukocyte (Figure 2C), but no significant differences were detected in levels of plasma NO in comparison with seronegative subjects (Figure 2D). The infection of peripheral blood from seronegative and seropositive patients with trypomastigotes (Tulahuen strain) significantly increased the percentage of NO- and ROS-producing leukocytes (Figure S2 in Supplementary Material).

\section{CD8+ T Lymphocytes from Chagasic Patients Exhibited High Levels of Nitrated Tyrosine Residues}

Non-chagasic individuals exhibited significantly lower tyrosine nitration (NT) of leukocytes, lymphocytes, and T cells than chagasic patients (Figure 3A). In particular, chagasic patients showed 


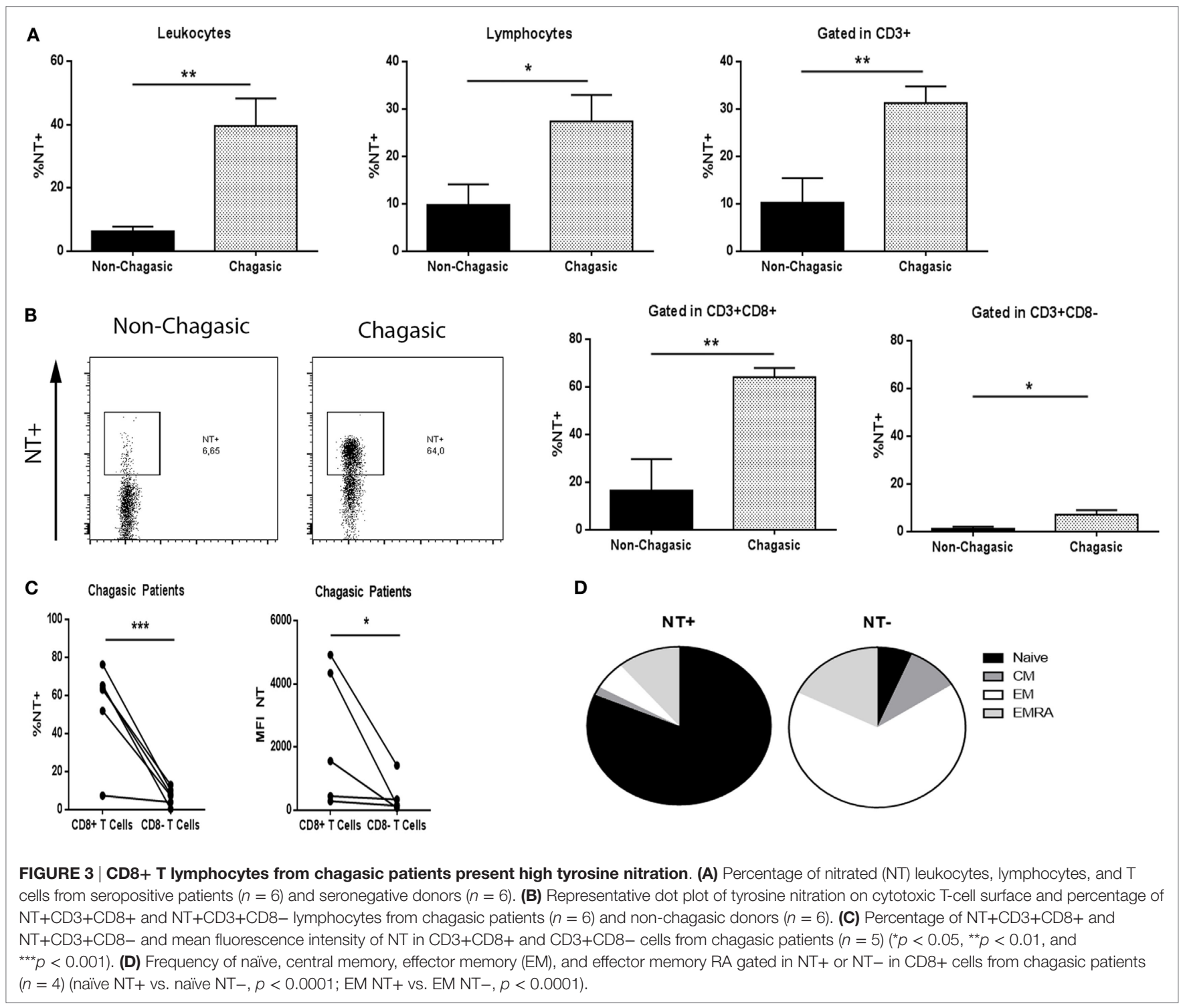

more NT+CD8+ T lymphocytes compared to non-chagasic donors (Figure 3B). Although nitration also increased in CD8$\mathrm{T}$ cell population from seropositive patients, a main effect was observed in CD8+ T lymphocytes compared to CD8- T cells in this group of patients (Figure $3 \mathrm{C}$ ). Among NT+ cytotoxic cells from Chagas patients, we have identified naïve cells as the main subpopulation that undergoes tyrosine nitration (NT+ vs. NT$81.45 \pm 3.00$ vs. $6.50 \pm 1.61 \%)$. On the contrary, the majority of $\mathrm{NT}-$ cells were EM CD8+ T cells $(\mathrm{NT}+$ vs. $\mathrm{NT}-5.73 \pm 1.22$ vs. $66.33 \pm 4.46 \%)$ (Figure 3D).

\section{Cytotoxic T Cells from Chagas Patients Were Less Functional}

Even though CD4+ T lymphocytes from seropositive and seronegative individuals showed similar levels of expression of the TCR $\zeta$ chain, the cytotoxic T cells exhibited a diminished amount of TCR $\zeta$ in chagasic patients, as compared to non-chagasic donors (Figure 4A). In line with these observations, CD8 T cell population from seropositive patients showed lower frequency of CD107a+ cells, as well as IFN $\gamma$, TNF, and IL-2-producing cells after stimulation with PMA/Ionomycin (unspecific stimuli) in comparison with the same population from seronegative donors (Figure 4B). Furthermore, after anti-CD3/anti-CD28 stimulation (via TCR), the percentage of CD8+ T cells from Chagas patients positive for CD107a, IFN $\gamma, \mathrm{TNF}$, and IL-2 was significantly diminished in NT+ cells compared to NT - cells (Figure 4C). The same behavior was observed when we compared the functionality of nitrated vs. no nitrated CD8+ T cells within the EM-EMRA populations (Figure S3 in Supplementary Material). These results indicated that nitrated cytotoxic T cells from chagasic patients were less functional than non-nitrated population. Moreover, chagasic patients showed a decreased percentage of CD39+ and CD73+ lymphocytes compared to control donors (Figure 4D). However, 
A

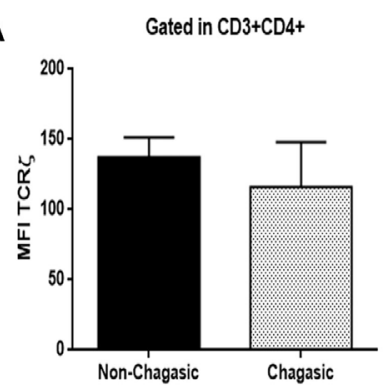

B

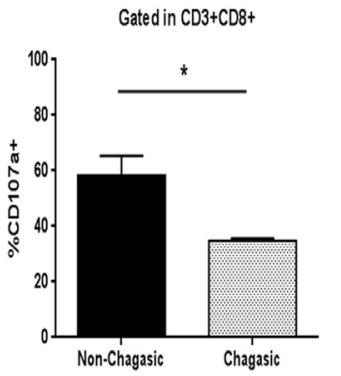

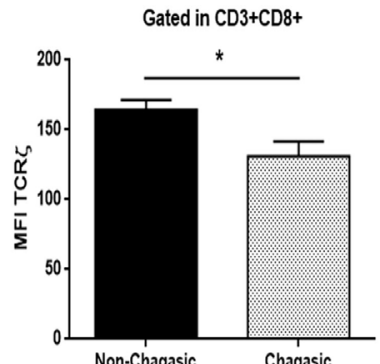
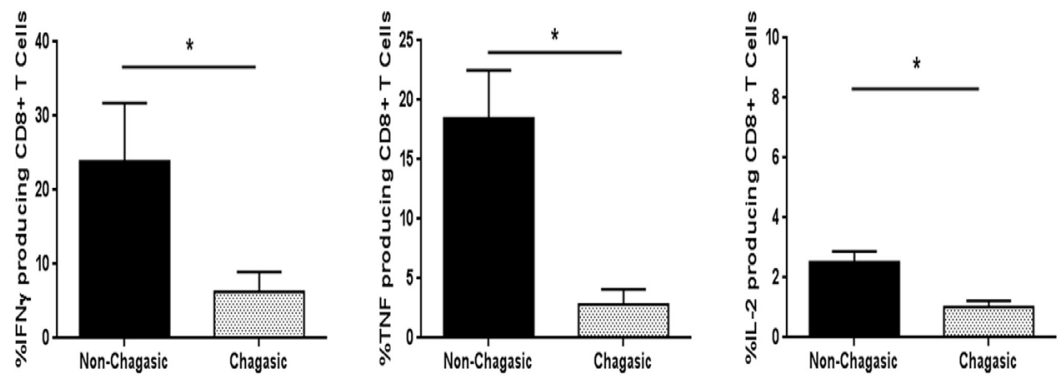

C
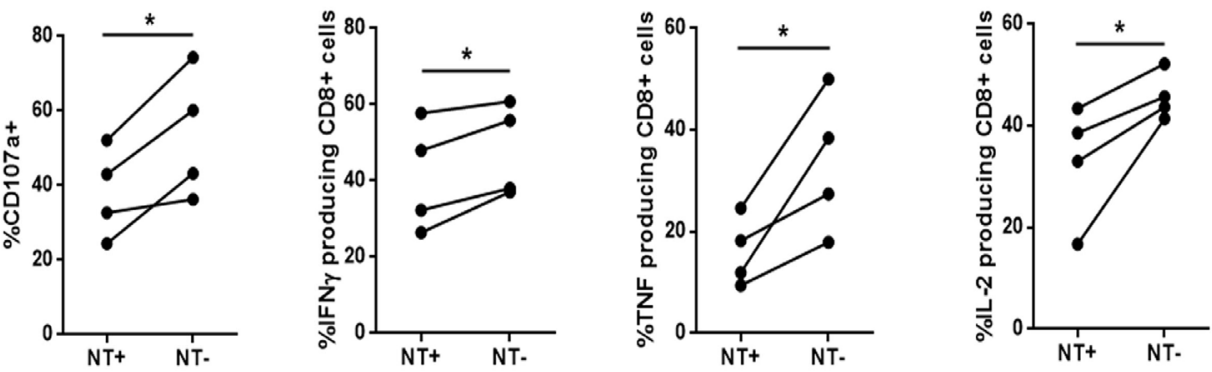

D
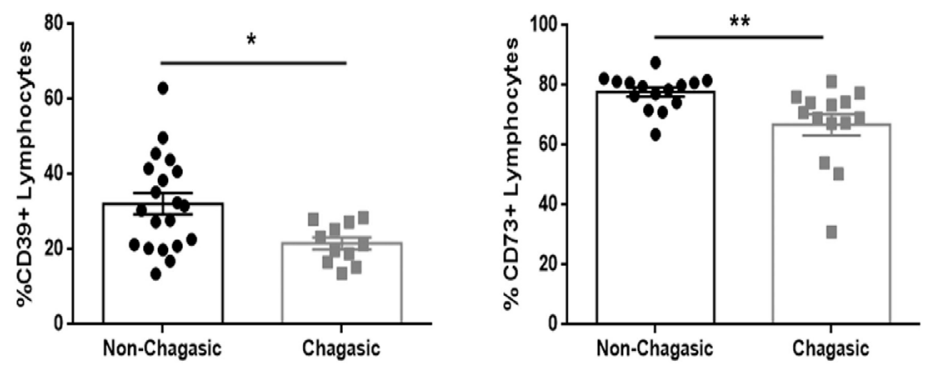

FIGURE 4 | Cytotoxic T cells from chagasic patients are less functional than those from healthy individuals. (A) Mean fluorescence intensity of TCR -chain in CD3+CD4+ and CD3+CD8+ cells; (B) frequency of CD107a+ cells, IFN $\gamma$, TNF, and IL-2-producing CD8+ T Iymphocytes from chagasic patients $(n=5)$ and seronegative donors $(n=5)$ after PMA/ionomycin stimulation. (C) Frequency of CD107a+ cells, IFN $\gamma$, TNF, and IL-2-producing NT+ and NT- gated in CD8+ T lymphocytes from chagasic patients $(n=4)$ after anti-CD3+ anti-CD28 stimulation. (D) Percentage of CD39+ and CD73+ leukocytes from seropositive $(n=20)$ and seronegative $(n=14)$ individuals $\left({ }^{*} p<0.05\right.$ and $\left.{ }^{* *} p<0.01\right)$.

frequency of CD8+ T cells expressing Tim-3, PD-1, or CTLA-4 was no different between chagasic patients and seronegative donors (Figure S4 in Supplementary Material). The results suggest that although cytotoxic $\mathrm{T}$ cells are less functional in seropositive subjects, they appear not to be a classically exhausted population since the expression of inhibitory receptors was not upregulated compared to CD8+ T cells from seronegative subjects.

\section{IL-1ß Plasma Levels Were Increased in Seropositive Patients}

The cytokine plasma levels were assayed using a panel of capture beads. In seronegative and seropositive individuals, the levels of IL-6, IFN $\gamma$, TNF, and IL-4 were similar. However, IL- $1 \beta$ levels were higher, and the amount of IL-10 was lower in plasma from chagasic people compared with control donors (Figure 5). 

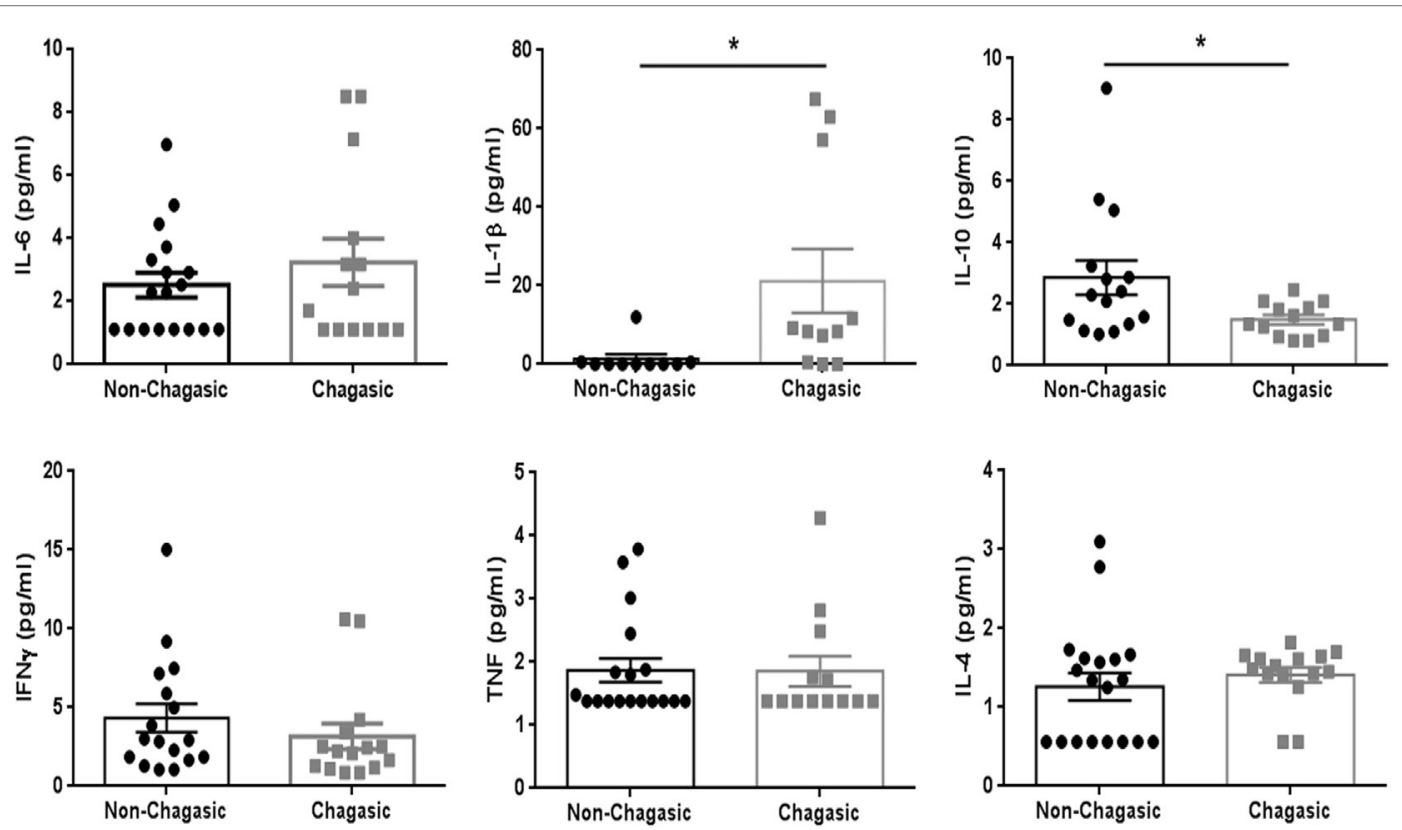

FIGURE 5 | IL-1 $\beta$ plasma levels are higher in seropositive patients than in seronegative donors. Plasma levels of IL-6, IL-10, IFN $\gamma$, TNF, and IL-4 (assayed by a panel of capture beads) and IL-1 $\beta$ (evaluated by enzyme-linked immunosorbent assay) in chagasic patients $(n=18)$ and non-chagasic donors $(n=15)\left({ }^{*} p<0.05\right)$

\section{IL-6 Prevented CD8+ T Cell Nitration and Apoptosis Susceptibility and Decreased IL-1 $\beta$ Production by Infected PBMCs}

The IL-6 stimulation of in vitro-infected PBMCs obtained from healthy donors blunted T. cruzi-induced NT in CD3+CD8+ cells. Conversely, the treatment of these cultures with a neutralizing antibody specific for IL-6 ( $\alpha$ IL-6) increased the percentage of T. cruzi-induced NT in this subpopulation (Figure 6A). Moreover, IL-6 treatment rescued infected CD8+ T cells from apoptosis (Figure 6B). Concomitantly, while IL-6 stimulation diminished the levels of IL-1 $\beta$ in culture supernatants from T-cruzi-infected PBMCs, $\alpha$ IL- 6 treatment increased the release of IL-1 $\beta$ (Figure 6C). Furthermore, although IL-6 stimulation did not affect NO production by T. cruzi-infected PBMCs, the blockage of IL-6 significantly increased T. cruzi-induced NO production (Figure 6D). Additionally, IL-6 stimulation diminished NT in infected CD3+CD8+ cells from peripheral blood of chagasic patients (Figure S5 in Supplementary Material). In accordance, we found that blocking IL- 6 and IL- $1 \beta$ significantly diminished the percentage of $\mathrm{NT}+\mathrm{CD} 8+\mathrm{T}$ cells from in vitro-infected PBMCs in comparison with cultures incubated with anti-IL-6 alone (Figure 7A). Moreover, the percentage of NO-producing monocytes significantly diminished when both cytokines were blocked compared with the inhibition of IL-6 alone (Figure 7B).

\section{DISCUSSION}

Multiple immunological effector mechanisms are critical for resolving T. cruzi infection, but considering that this parasite invades and replicates in essentially all types of mammalian cells, $\mathrm{T}$ cells and monocytes/macrophages are particularly important for controlling the infection. As with other intracellular parasites, T. cruzi antigens are processed and presented on MHC-class I molecules, leading to the recognition of parasite components by CD8+ T cells. In this regard, the study of the induction of CD8+ T cell-mediated protective immunity has become a center of intense research efforts to find control measures and prophylactic tools that could be used to produce effective therapeutic vaccines. This study shows that chronic T. cruzi infection leads to a significant nitration of T lymphocytes, mainly of the CD8+ $\mathrm{T}$ cell subset. The increased tyrosine nitration was associated with impaired effector functions and a significant fall in the number and percentage of circulating CD8 $+\mathrm{T}$ cells in chronic Chagas patients.

Among the reactive oxygen and nitrogen species mediating T-cell suppression is peroxynitrite, one of the most potent oxidants in the body. The hyperproduction of peroxynitrite is associated with nitration of the surface proteins in T cells $(11,12)$. Regarding CD8+ T cells, nitration of tyrosines within the TCR/ CD8 complex disrupts the binding of the specific peptide-MHC dimers to CD8 molecules, which results in the inability of this T cell subset to bind MHC $(18,19)$. Furthermore, peroxynitrites also inhibit TCR signaling by preventing the association of CD3$\zeta$ with the TCR. Our group has reported that T. cruzi induces an increase in splenic NT+CD8+ and NT+CD4+ T cells from infected mice by performing confocal and flow cytometric analysis of immunofluorescence staining (13). In this work, we found that the increased nitration of CD8+ T cells was associated with a lower capacity for activation (TCR $\zeta$ expression), a 

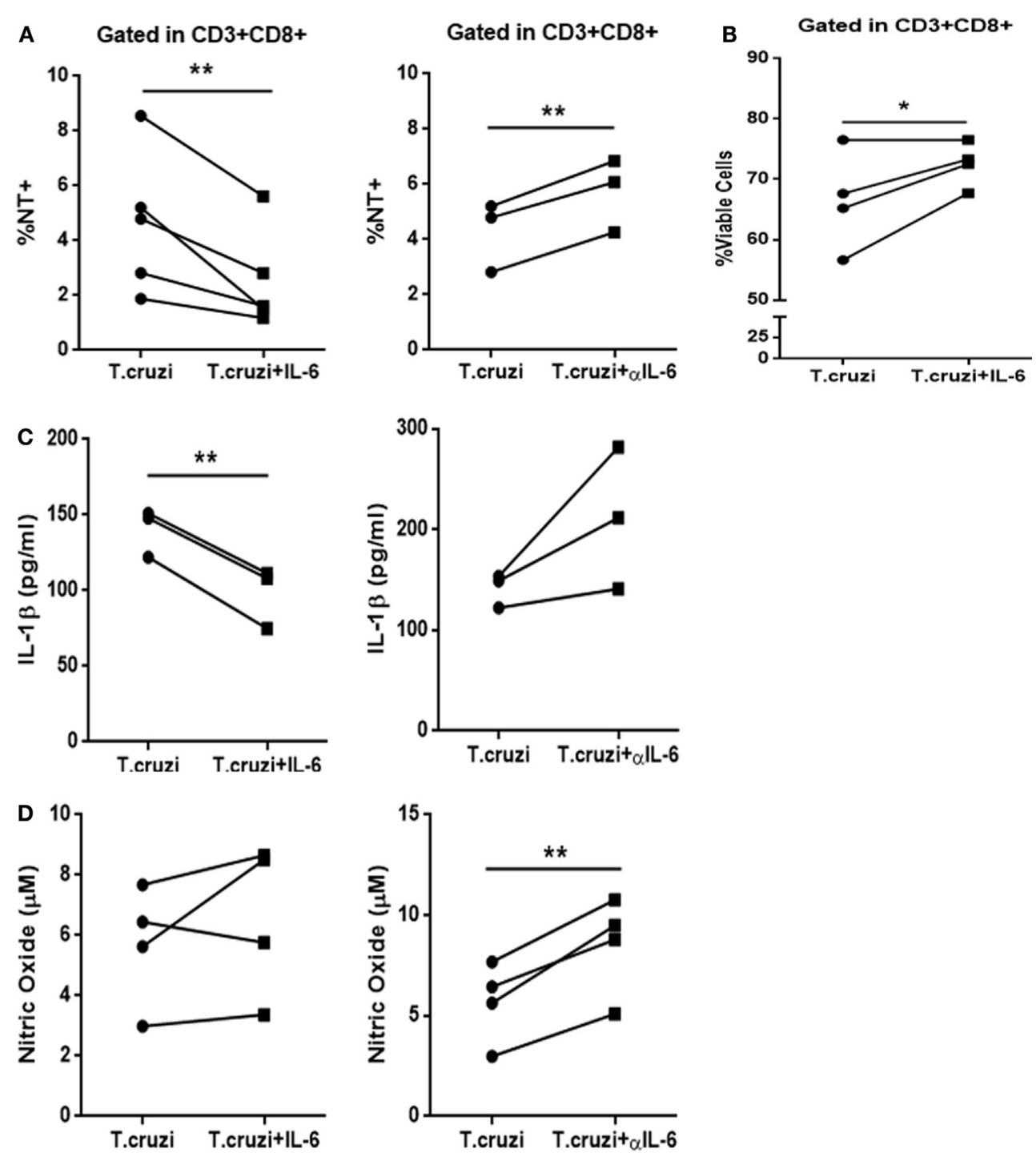

FIGURE 6 | IL-6 prevents nitration, increases survival of CD3+CD8+ cells, and decreases IL-1 $\beta$ levels after infection. (A) Percentage of NT+CD3+CD8+ cells in infected peripheral blood mononuclear cells (PBMCs) from non-chagasic $(n=5)$ donors after 48 h of stimulation with IL-6 (Trypanosoma cruzi + IL-6) or $\alpha \mathrm{LL}-6$ (T. cruzi $+\alpha \mathrm{lL}-6)$ or medium alone (T. cruzi). (B) Percentage of viable CD3+CD8+ cells infected with T. cruzi (T. cruzi) or infected and stimulated with IL-6

(T. cruzi + IL-6). (C) IL-1 $\beta$ levels and (D) nitric oxide levels in culture supernatants of infected PBMCs after 48 h of stimulation with IL-6 (T. cruzi + IL-6) or $\alpha \mathrm{IL}-6$ (T. cruzi $+\alpha \mid \mathrm{L}-6)$ or medium alone $\left(\right.$ T. cruzi) $\left({ }^{*} p<0.05\right.$ and $\left.{ }^{\star *} p<0.01\right)$.

diminished production of cytokines (IFN $\gamma$, TNF, and IL-2) and deactivation of cytotoxic functions (CD107a expression) in this cell population. Strikingly, cellular nitration seems to be reversible, since the incubation of infected leukocytes from seropositive patients with IL-6 significantly diminished the percentage of NT+ lymphocytes.

Leukocytes from chagasic patients significantly increased the production of NO, which correlated with increased susceptibility of CD8+ T cells to undergo spontaneous apoptosis. NO-mediated suppression of T-cell activation does not seem to be mediated by events triggered by TCR recognition but, instead, with the signaling cascade that is downstream of IL-2 (20). In human
T cells, NO affects the stability of IL-2 mRNA and the release of IL-2. In this sense, NO negatively regulates intracellular signaling proteins either directly, by S-nitrosylation of crucial cysteine residues, or indirectly, by activation of cyclic-GMP-dependent protein kinase (21). Furthermore, while NO also sensitizes cells to Fas-L-mediated apoptosis (22), the anti-apoptotic molecule Bcl-2 diminished the susceptibility to NO-induced apoptosis (23). In line with these reports, we found that CD8+ T cells from seropositive individuals showed a significantly decreased expression of $\mathrm{Bcl}-2$ concomitant with the significant diminution in their viability. The increased susceptibility to apoptosis was in accordance with the diminution in the percentage as well as 

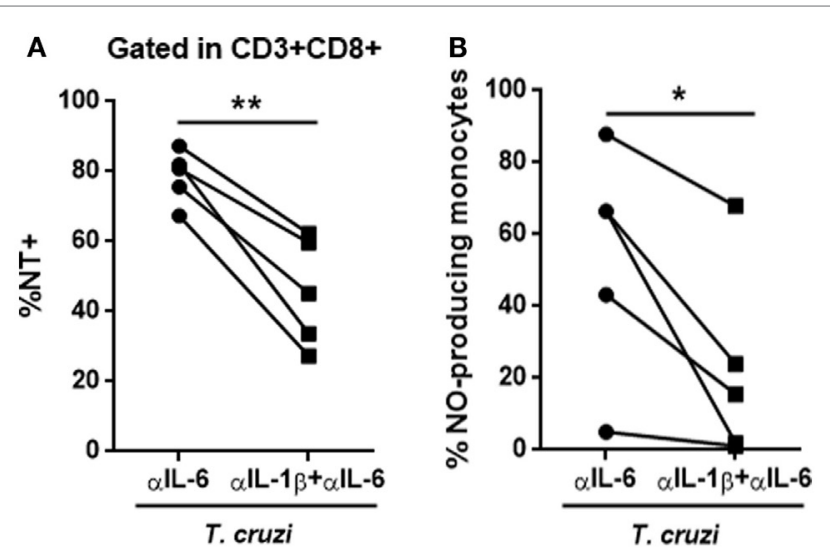

FIGURE 7 | The blockage of IL-6 and IL-1 $1 \beta$ reverts the phenotype induced by anti-IL-6. (A) Percentage of NT+CD8+ T cells and (B) NO-producing monocytes (CD14+) in in vitro-infected peripheral blood mononuclear cells from seronegative $(n=5)$ subjects after $48 \mathrm{~h}$ of stimulation with anti-IL-6 or anti-IL- $6+$ anti-IL-1 $\beta\left({ }^{*} p<0.05\right.$ and $\left.{ }^{* *} p<0.01\right)$.

absolute number of total $\mathrm{T}$ cells at the expense of diminution of $\mathrm{CD} 8+\mathrm{T}$ cells but not of $\mathrm{T}$ helper cells or $\mathrm{B}$ cells.

It was reported that NO levels, as measured by Griess reagent assay, were not significantly increased in the plasma of seropositive subjects compared with those in seronegative samples. However, the plasma level of 3-nitrotyrosine (NT), was increased in seropositive subjects (24). The results indicated that seropositive subjects with Chagas disease are exposed to increased nitrosative stress. In total agreement with this report, we observed no significant increase in plasma levels of nitrate/nitrite of seropositive subjects compared with seronegative donors. Nevertheless, the leukocytes from chagasic patients significantly increased the production of NO, suggesting that $\mathrm{NO}$ produced by leukocytes, rather than soluble NO, may contribute to increased nitrosative stress in patients with Chagas disease, evidencing the importance of cell-to-cell contact for NO inhibitory effector functions $(25,26)$. Although tyrosine nitration constitutes one of the mechanisms employed by MDSC to suppress T cell response through cell-to-cell contact, it may also be dependent on the activity of myeloperoxidase, secreted by monocytes and polymorphonuclear neutrophils (27).

In accordance with our recent observations, while IL-1 $\beta$ plasma levels were significantly increased in chagasic patients, the stimulation of in vitro-infected PBMCs with IL-6 significantly diminished the production of IL-1 $\beta$. Moreover, IL- 6 diminished the nitration rate and improved the survival of CD8+ T cells. In agreement with this result, we have previously shown that IL-6 acts as a survival factor for infected target cells $(28,29)$. We have observed that IL- 6 regulates IL- $1 \beta$-induced NO production, and that excessive oxidative stress accounts for the increased mortality of T. cruzi-infected IL6KO mice (unpublished results). The anti-inflammatory action of IL-6 appears to be central to controlling cardiac and systemic oxidative stress, promoting cellular rescue against apoptosis, and protecting infected IL-6-deficient mice against death. In this model, this was clearly illustrated by the fact that the percentage of nitrated CD8+ T cells observed after blocking IL- 6 was significantly diminished when anti-IL-6 was combined with anti-IL-1 $\beta$. In accordance, IL-6 stimulation of peripheral blood cells from chagasic patients induced a significant diminution of nitration of CD8+ T cells. Our results strongly suggest that IL- 6 could represent a key factor for regulating the nitration of proteins on human cytotoxic T cells.

One potential regulatory system that could have a role in the IL-6-induced anti-inflammatory effects is the extracellular levels of adenosine. T. cruzi infection of cardiac tissue induces an influx of immune cells that consume large quantities of oxygen. Ischemic cells rapidly respond to the hypoxic and inflammatory environment by releasing ATP (normally present within cardiomyocytes in millimolar concentrations). Once in the extracellular environment, ATP is converted to AMP and then to adenosine by the CD39 and CD73 ectoenzymes, respectively (30). In pathological conditions, high levels of ATP act as a proinflammatory danger signal, activating the inflammasome that processes pro-IL-1 $\beta$ into mature IL- $1 \beta(31,32)$. In this sense, it has been suggested that CD39 expression may contribute to dampening the ongoing inflammatory processes and/or rescue the cells from ATP-induced apoptosis/necrosis (33). Ultimately, adenosine exerts potent anti-inflammatory effects on different immune cell types. Recently, we reported that purinergic signaling is clue in regulating the immune response to experimental T. cruzi infection. The temporal pharmacological inhibition of CD73 during the early acute phase of the infection induces microbicidal mechanisms, with the concomitant reduction in cardiac parasite load, improving the outcome of chronic cardiomyopathy (34). In agreement with a previous report that indicated CD73 expression on T cells is downregulated in chronic HIV infection (35), in this work, we observed lower expression of CD39 and CD73 on fresh explanted lymphocytes from chagasic patients compared to seronegative donor. Thus, it is plausible that the downregulation of ATP catabolic enzymes could be involved in the increased plasma IL-1 $\beta$ levels in chagasic patients.

In summary, the results of this study show that chronic T. cruzi infection leads to a decrease in the number and percentage of total circulating CD8+ T cells and that NO produced by leukocytes may contribute to lowering the effector function of these cells, which ultimately may results in an inefficient control of parasite replication. The findings also suggest that IL- 6 could be a key factor to improve CD8+ T cell activation and survival.

\section{AUTHOR CONTRIBUTIONS}

Conceived and designed the experiments: LS, MR, and MA. Performed the experiments: LS, LV, NE, MR, and NP. Analyzed the data: LS, LV, NE, MR, NP, NS, MV, GB, SG, AM, and MA. Patients handling and human samples: LV, NS, MV, GB, and AM. Wrote the paper: LS and MA.

\section{ACKNOWLEDGMENTS}

The authors thank Laura Gatica, Alejandra Romero, Pilar Crespo, and Paula Abadie for their skillful technical assistance. They also 
thank the staff of the Biochemical Laboratory of Diagnosis of the HNSM for their kind collaboration. MA and SG are members of the scientific career from the Consejo Nacional de Investigaciones Científicas y Técnicas de la República Argentina (CONICET). LS, $M R$, and NP thank CONICET for the fellowships granted; LV and NE thank fellowship granted from Fundación Florencio Fiorini and ANPCyT-FONCyT, respectively.

\section{FUNDING}

This work was supported by Secretaría de Ciencia y Tecnología, Universidad Nacional de Córdoba (Grant number 05/C642),

\section{REFERENCES}

1. Tarleton RL. CD8+ T cells in Trypanosoma cruzi infection. Semin Immunopathol (2015) 37(3):233-8. doi:10.1007/s00281-015-0481-9

2. Savino W, Villa-Verde DM, Mendes-da-Cruz DA, Silva-Monteiro E, Perez AR, Aoki Mdel P, et al. Cytokines and cell adhesion receptors in the regulation of immunity to Trypanosoma cruzi. Cytokine Growth Factor Rev (2007) 18(1-2):107-24. doi:10.1016/j.cytogfr.2007.01.010

3. Burgos JM, Diez M, Vigliano C, Bisio M, Risso M, Duffy T, et al. Molecular identification of Trypanosoma cruzi discrete typing units in end-stage chronic Chagas heart disease and reactivation after heart transplantation. Clin Infect Dis (2010) 51(5):485-95. doi:10.1086/655680

4. Aoki MP, Guinazu NL, Pellegrini AV, Gotoh T, Masih DT, Gea S. Cruzipain, a major Trypanosoma cruzi antigen, promotes arginase-2 expression and survival of neonatal mouse cardiomyocytes. Am J Physiol Cell Physiol (2004) 286(2):C206-12. doi:10.1152/ajpcell.00282.2003

5. Aoki MP, Carrera-Silva EA, Cuervo H, Fresno M, Girones N, Gea S. Nonimmune cells contribute to crosstalk between immune cells and inflammatory mediators in the innate response to Trypanosoma cruzi infection. J Parasitol Res (2012) 2012:13. doi:10.1155/2012/737324

6. Mabbott NA, Sutherland IA, Sternberg JM. Suppressor macrophages in Trypanosoma brucei infection: nitric oxide is related to both suppressive activity and lifespan in vivo. Parasite Immunol (1995) 17(3):143-50. doi:10.1111/ j.1365-3024.1995.tb01016.x

7. Jenson JS, O'Connor R, Osborne J, Devaney E. Infection with Brugia microfilariae induces apoptosis of CD4(+) T lymphocytes: a mechanism of immune unresponsiveness in filariasis. Eur J Immunol (2002) 32(3):858-67. doi:10.1002/1521-4141(200203)32:3<858::AID-IMMU858>3.0.CO;2-E

8. Rockett KA, Awburn MM, Rockett EJ, Cowden WB, Clark IA. Possible role of nitric oxide in malarial immunosuppression. Parasite Immunol (1994) 16(5):243-9. doi:10.1111/j.1365-3024.1994.tb00346.x

9. Saio M, Radoja S, Marino M, Frey AB. Tumor-infiltrating macrophages induce apoptosis in activated $\mathrm{CD} 8(+) \mathrm{T}$ cells by a mechanism requiring cell contact and mediated by both the cell-associated form of TNF and nitric oxide. J Immunol (2001) 167(10):5583-93. doi:10.4049/jimmunol.167. 10.5583

10. Hirst DG, Robson T. Nitrosative stress as a mediator of apoptosis: implications for cancer therapy. Curr Pharm Des (2010) 16(1):45-55. doi:10.2174/ 138161210789941838

11. Sawa $\mathrm{T}$, Akaike $\mathrm{T}$, Maeda $\mathrm{H}$. Tyrosine nitration by peroxynitrite formed from nitric oxide and superoxide generated by xanthine oxidase. J Biol Chem (2000) 275(42):32467-74. doi:10.1074/jbc.M910169199

12. Gunaydin H, Houk KN. Mechanisms of peroxynitrite-mediated nitration of tyrosine. Chem Res Toxicol (2009) 22(5):894-8. doi:10.1021/tx800463y

13. Arocena AR, Onofrio LI, Pellegrini AV, Carrera-Silva AE, Paroli A, Cano RC, et al. Myeloid-derived suppressor cells are key players in the resolution of inflammation during a model of acute infection. Eur J Immunol (2014) 44(1):184-94. doi:10.1002/eji.201343606

14. Albareda MC, Laucella SA, Alvarez MG, Armenti AH, Bertochi G, Tarleton RL, et al. Trypanosoma cruzi modulates the profile of memory CD8+ $\mathrm{T}$ cells in chronic Chagas' disease patients. Int Immunol (2006) 18(3):465-71. doi:10.1093/intimm/dxh387
Agencia Nacional de Promoción Científica y Tecnológica (ANPCyT), Fondo para la Investigación Científica y Tecnológica (PICT 2013-2885), Consejo Nacional de Investigaciones Científicas y Técnicas (CONICET) (PIP 11220120100620), and by Ministerio de Ciencia y Tecnología, Gobierno de la Provincia de Córdoba (1143/10).

\section{SUPPLEMENTARY MATERIAL}

The Supplementary Material for this article can be found online at http://journal.frontiersin.org/article/10.3389/fimmu. 2016.00626/full\#supplementary-material.

15. Sallusto F, Geginat J, Lanzavecchia A. Central memory and effector memory T cell subsets: function, generation, and maintenance. Annu Rev Immunol (2004) 22:745-63. doi:10.1146/annurev.immunol.22.012703.104702

16. Ding AH, Nathan CF, Stuehr DJ. Release of reactive nitrogen intermediates and reactive oxygen intermediates from mouse peritoneal macrophages. Comparison of activating cytokines and evidence for independent production. J Immunol (1988) 141:2407-12.

17. Plan.Nacional.de.Chagas. Plan Nacional de Chagas 2011-2015. Boletín Oficial Junio - Res 867/2012. Argentina: Official Bulletin of National Ministry of Health (2012).

18. Nagaraj S, Gupta K, Pisarev V, Kinarsky L, Sherman S, Kang L, et al. Altered recognition of antigen is a mechanism of CD8+ T cell tolerance in cancer. Nat Med (2007) 13(7):828-35. doi:10.1038/nm1609

19. Nagaraj S, Schrum AG, Cho HI, Celis E, Gabrilovich DI. Mechanism of T cell tolerance induced by myeloid-derived suppressor cells. J Immunol (2010) 184(6):3106-16. doi:10.4049/jimmunol.0902661

20. Mazzoni A, Bronte V, Visintin A, Spitzer JH, Apolloni E, Serafini P, et al. Myeloid suppressor lines inhibit T cell responses by an NO-dependent mechanism. J Immunol (2002) 168(2):689-95. doi:10.4049/jimmunol.168.2.689

21. Bronte V, Zanovello P. Regulation of immune responses by L-arginine metabolism. Nat Rev Immunol (2005) 5(8):641-54. doi:10.1038/nri1668

22. Bonavida B, Garban H. Nitric oxide-mediated sensitization of resistant tumor cells to apoptosis by chemo-immunotherapeutics. Redox Biol (2015) 6:486-94. doi:10.1016/j.redox.2015.08.013

23. Messmer UK, Reed UK, Brune B. Bcl-2 protects macrophages from nitric oxide-induced apoptosis. J Biol Chem (1996) 271(33):20192-7. doi:10.1074/ jbc.271.33.20192

24. Dhiman M, Estrada-Franco JG, Pando JM, Ramirez-Aguilar FJ, Spratt H, Vazquez-Corzo $\mathrm{S}$, et al. Increased myeloperoxidase activity and protein nitration are indicators of inflammation in patients with Chagas' disease. Clin Vaccine Immunol (2009) 16(5):660-6. doi:10.1128/CVI.00019-09

25. Gabrilovich DI, Nagaraj S. Myeloid-derived suppressor cells as regulators of the immune system. Nat Rev Immunol (2009) 9(3):162-74. doi:10.1038/ nri2506

26. Lu T, Gabrilovich DI. Molecular pathways: tumor-infiltrating myeloid cells and reactive oxygen species in regulation of tumor microenvironment. Clin Cancer Res (2012) 18(18):4877-82. doi:10.1158/1078-0432.CCR-11-2939

27. Predonzani A, Cali B, Agnellini AH, Molon B. Spotlights on immunological effects of reactive nitrogen species: when inflammation says nitric oxide. World J Exp Med (2015) 5(2):64-76. doi:10.5493/wjem.v5.i2.64

28. Ponce NE, Cano RC, Carrera-Silva EA, Lima AP, Gea S, Aoki MP. Toll-like receptor- 2 and interleukin- 6 mediate cardiomyocyte protection from apoptosis during Trypanosoma cruzi murine infection. Med Microbiol Immunol (2012) 201(2):145-55. doi:10.1007/s00430-011-0216-Z

29. Ponce NE, Carrera-Silva EA, Pellegrini AV, Cazorla SI, Malchiodi EL, Lima AP, et al. Trypanosoma cruzi, the causative agent of Chagas disease, modulates interleukin-6-induced STAT3 phosphorylation via gp130 cleavage in different host cells. Biochim Biophys Acta (2013) 1832(3):485-94. doi:10.1016/ j.bbadis.2012.12.003

30. Antonioli L, Pacher P, Vizi ES, Hasko G. CD39 and CD73 in immunity and inflammation. Trends Mol Med (2013) 19(6):355-67. doi:10.1016/ j.molmed.2013.03.005 
31. Lamkanfi M, Dixit VM. Mechanisms and functions of inflammasomes. Cell (2014) 157(5):1013-22. doi:10.1016/j.cell.2014.04.007

32. Garg NJ. Inflammasomes in cardiovascular diseases. Am J Cardiovasc Dis (2011) 1(3):244-54.

33. Zhou Q, Yan J, Putheti P, Wu Y, Sun X, Toxavidis V, et al. Isolated CD39 expression on CD4+ T cells denotes both regulatory and memory populations. Am J Transplant (2009) 9(10):2303-11. doi:10.1111/j.1600-6143.2009.02777.x

34. Ponce NE, Sanmarco LM, Eberhardt N, Garcia MC, Rivarola HW, Cano RC, et al. CD73 inhibition shifts cardiac macrophage polarization toward a microbicidal phenotype and ameliorates the outcome of experimental Chagas cardiomyopathy. J Immunol (2016) 197(3):814-23. doi:10.4049/ jimmunol.1600371

35. Toth I, Le AQ, Hartjen P, Thomssen A, Matzat V, Lehmann C, et al. Decreased frequency of CD73+CD8+ T cells of HIV-infected patients correlates with immune activation and T cell exhaustion. J Leukoc Biol (2013) 94(4):551-61. doi:10.1189/jlb.0113018
Conflict of Interest Statement: The authors declare no competing financial interests. They did not receive payment or services from a third part for any aspect of the submitted work.

The reviewer CL and handling Editor declared their shared affiliation, and the handling Editor states that the process nevertheless met the standards of a fair and objective review.

Copyright $\odot 2016$ Sanmarco, Visconti, Eberhardt, Ramello, Ponce, Spitale, Vozza, Bernardi, Gea, Minguez and Aoki. This is an open-access article distributed under the terms of the Creative Commons Attribution License (CC BY). The use, distribution or reproduction in other forums is permitted, provided the original author(s) or licensor are credited and that the original publication in this journal is cited, in accordance with accepted academic practice. No use, distribution or reproduction is permitted which does not comply with these terms. 